\title{
Técnicas de Projeção para Identificação de Grupos e Comparação de Dados Multidimensionais Usando Diferentes Medidas de Similaridade
}

\author{
Paulo Joia $^{1}$, Luis Gustavo Nonato ${ }^{1}$ \\ ${ }^{1}$ Instituto de Ciências Matemáticas e de Computação (ICMC) - USP, São Carlos, Brasil \\ \{pjoia, gnonato\} @icmc.usp.br
}

\begin{abstract}
This work explores the potential of the projection techniques to solve problems related to: clustering and similarity search in multidimensional data. For clustering data, a local and interactive projection technique capable of projecting data with effective preservation of distances was developed and also a new clustering method, which operates in the visual space, ensuring that clusters are not fragmented during the visualization. For the similarity search, we build a family of class-specific metrics and the fuzzy set theory was used to estimate a degree of uncertainty that is embedded in the metric, increasing its precision. The results confirm the effectiveness of the developed techniques, which represent significant contributions for this investigation area.
\end{abstract}

Resumo. Este trabalho explora o potencial das técnicas de projeção para resolver problemas relacionados à: identificação de agrupamentos e busca por similaridade em dados multidimensionais. Para identificação de agrupamentos foi desenvolvida uma técnica de projeção local e interativa capaz de projetar dados com ótima preservação de distâncias, além de um novo método para identificação de agrupamentos, o qual opera no espaço visual, garantindo que os grupos obtidos não fiquem fragmentados durante a visualização. Para as buscas por similaridade em dados multidimensionais, uma família de métricas baseada em classes foi construída e a teoria dos conjuntos fuzzy foi usada para estimar um valor de incerteza que é transferido para a métrica, aumentando sua precisão. Os resultados confirmam a efetividade das técnicas desenvolvidas, as quais representam significativa contribuição nesta área de investigação.

\section{Introdução}

Visualização de informação desempenha um papel importante na organização e exploração de dados multidimensionais, graças à capacidade de percepção visual do sistema cognitivo humano, assim como técnicas de projeção, as quais reduzem a dimensionalidade do conjunto de dados permitindo visualizar informações muitas vezes ocultas na alta dimensão. Este trabalho explora o potencial das técnicas de visualização de informação com ênfase em projeção para auxiliar na identificação de agrupamentos e busca por similaridade em dados multidimensionais.

\subsection{Motivação}

Consideráveis avanços têm sido observados nas técnicas de projeção nos últimos anos, com aplicações em diferentes domínios. Algumas até propõem soluções para os problemas de identificação de agrupamentos e busca por similaridade em dados multidimensionais, contudo, longe da solução ideal. Por exemplo, identificar grupos é uma tarefa 
complexa e na maioria das vezes os grupos obtidos não correspondem à verdadeira natureza dos dados, normalmente organizados com base na geometria. Quanto à busca por similaridade, existem várias abordagens, todavia, poucas empregam medidas de similaridade realmente aptas a discriminar objetos de acordo com as classes existentes.

\section{Contribuições}

A tese de doutorado aqui apresentada, produziu resultados significativos na área de visualização de informação, com ênfase em projeção multidimensional. Resultados que podem ser comprovados pelas novas técnicas desenvolvidas e respectivas metodologias empregadas no desenvolvimento, conforme sumarizado abaixo. Para um exame mais detalhado sobre cada técnica, incluindo sua formulação matemática, algoritmo, resultados e comparações com outras técnicas, recomendamos que o leitor consulte [Joia 2015].

\subsection{Técnica de Projeção Local Interativa}

Local Affine Multidimensional Projection (LAMP) [Joia et al. 2011a] permite manipular pontos de controle no espaço visual de modo a organizá-los, possibilitando ao usuário guiar a projeção, porém, com uma grande vantagem sobre as demais técnicas que se apoiam em subconjunto de amostras: requer um número muito reduzido de pontos de controle como entrada, tornando-se ideal para aplicações interativas. Tem formulação matemática baseada em mapeamentos ortogonais, garantindo ótima preservação de distâncias durante a projeção multidimensional e, não depende de grafos de vizinhança para construir o mapeamento. É altamente precisa, com baixo custo computacional, apropriada para aplicações interativas envolvendo grandes volumes de dados. LAMP está atualmente entre as técnicas do estado da arte em relação à preservação de distâncias e eficiência computacional, além de permitir projetar dados explorando tanto relações globais como locais entre instâncias, de maneira efetiva. A Figura 1 mostra o potencial da LAMP ao estabelecer uma correlação visual entre conjuntos de dados, a princípio, sem qualquer conexão.

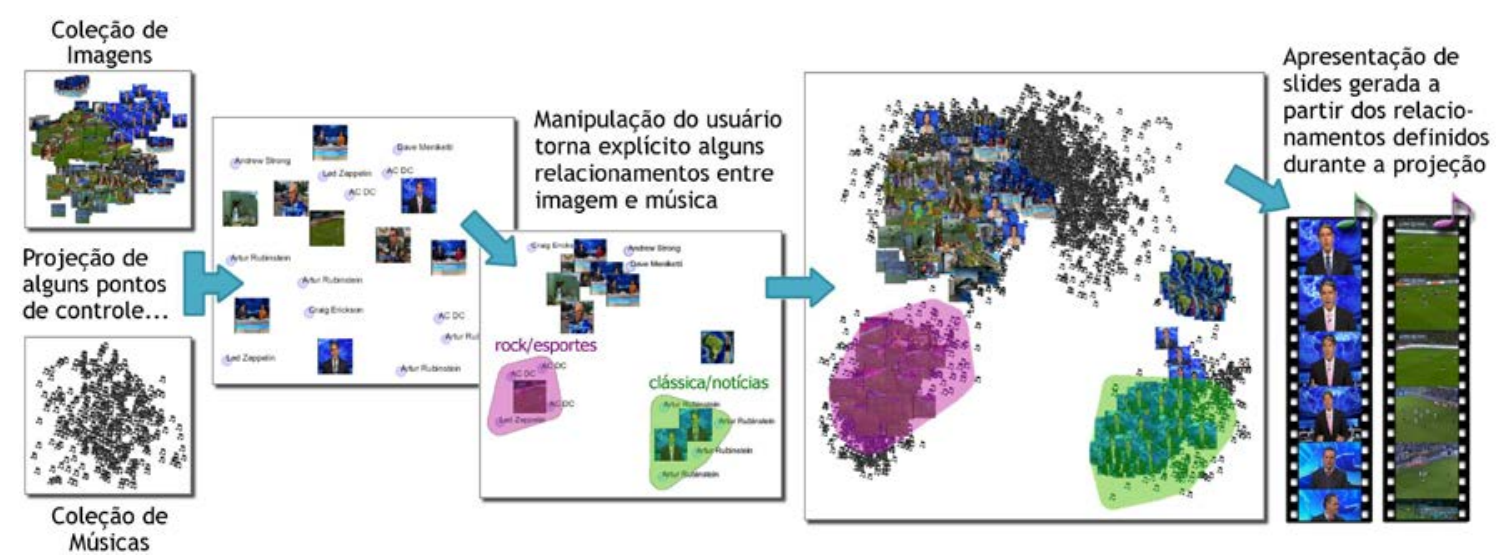

Figura 1. Utilizando a LAMP para correlacionar dados de diferentes naturezas. Inicialmente, uma projeção é criada para cada conjunto de dados, a partir de algumas amostras. A correlação entre as amostras é definida pelo usuário, agrupando objetos no espaço visual (imagens e músicas). Em seguida, os dados são projetados segundo as associações criadas pelo usuário. Por fim, as listas de objetos associados são usadas para criar uma apresentação de slides onde imagens e músicas são reproduzidas de forma sincronizada. 


\subsection{Método para Identificação de Grupos com Base em Projeção}

Column Selection Method (CSM) [Joia et al. 2015], um método de visualização apoiado em projeção multidimensional que permite agrupar dados. CSM opera no espaço visual, garantindo que os grupos obtidos não fiquem fragmentados durante a visualização. É orientado por um mecanismo de amostragem determinístico, capaz de identificar instâncias representativas que correspondem a um certo padrão nos dados. O mecanismo de amostragem é baseado em decomposição matricial (SVD) e capaz de operar mesmo em conjuntos de dados desbalanceados. Além de identificar instâncias representativas, o mecanismo de amostragem pode ser ajustado para identificar os atributos mais relevantes de cada agrupamento obtido. Portanto, em um único framework, três tarefas são contempladas: amostragem de dados, detecção de agrupamentos e seleção de atributos. A Figura 2 ilustra a metáfora visual utilizada pela CSM para representar grupos e atributos, por meio de superfícies e nuvens de palavras.

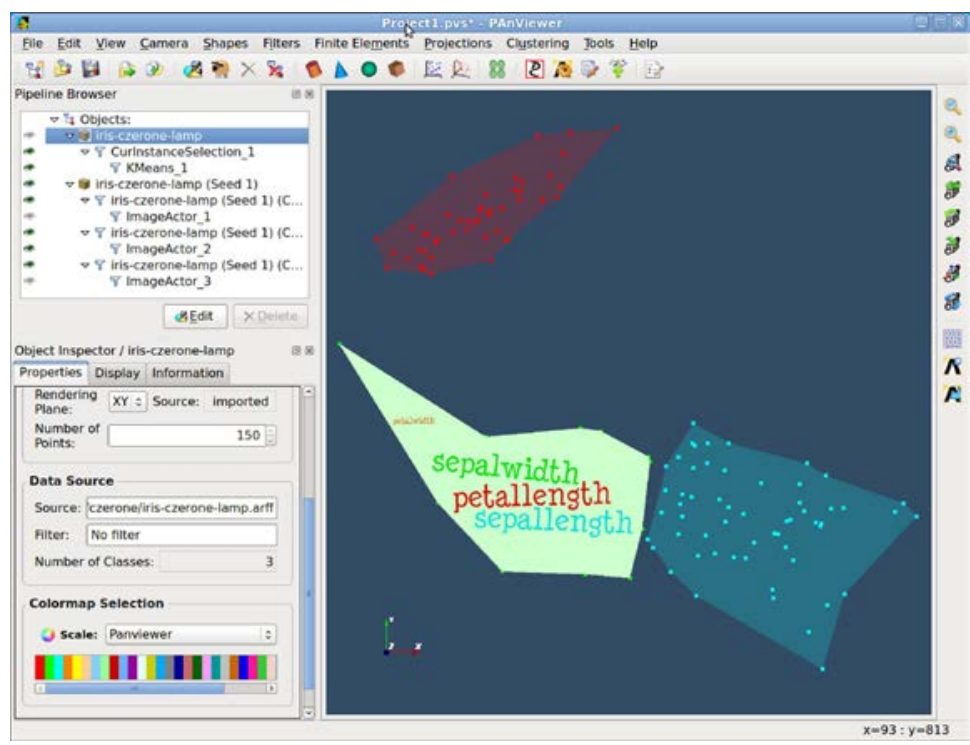

Figura 2. Metáfora visual utilizada pela CSM para representar grupos e atributos.

\subsection{Família de Métricas Classes-Específicas}

Muitas técnicas propõem medidas de similaridade para comparar dados multidimensionais, mas nenhuma diretamente relacionada às classes de objetos existentes no conjunto de dados. A Class-Specific Multidimensional Projection (CSMP) [Joia et al. 2012] é uma técnica de projeção baseada em uma família de métricas específicas por classe para projetar e comparar dados multidimensionais. As métricas são obtidas pela seleção dos atributos que melhor representam cada classe do conjunto de dados, de modo a minimizar a dissimilaridade entre pares de objetos pertencentes à mesma classe e, ao mesmo tempo, maximizá-la para objetos pertencentes a classes distintas. As métricas classes-específicas são avaliadas no contexto de recuperação de imagens por conteúdo para encontrar imagens similares a uma dada imagem de consulta. A lista de imagens similares pode ser retornada pelo sistema ou selecionada diretamente pelo usuário, a partir do layout da projeção, conforme exemplificado na Figura 3. 


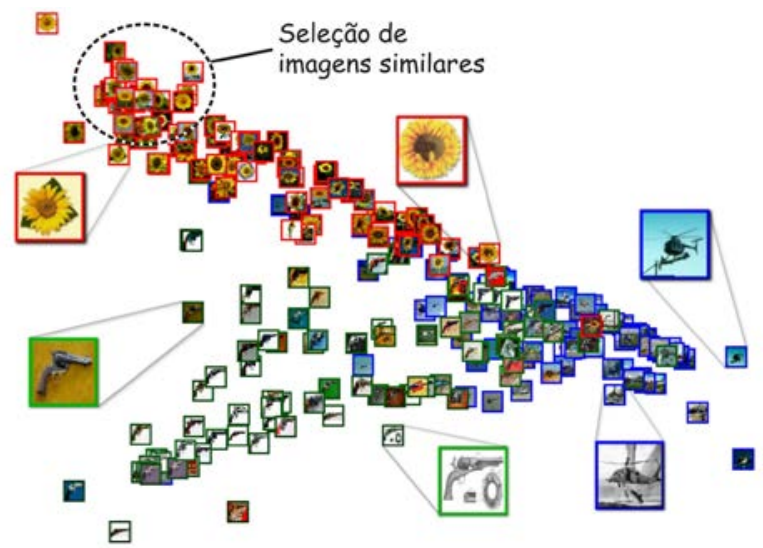

Figura 3. Seleção de imagens similares com o uso da CSMP.

\subsection{Cálculo de Incerteza na Família de Métricas Classes-Específicas}

Com o intuito de aumentar a precisão da família de métricas classes-específicas empregada na CSMP, uma nova técnica denominada Class-Specific with Weight Image Retrieval (CSWIRe) foi desenvolvida. Nesta abordagem, o usuário constrói um modelo a partir de um subconjunto de imagens, denominado "modelo de classes". A seguir, um classificador é aplicado sobre este modelo, retornando as melhores características e pesos que representam cada classe do modelo. Utilizando a teoria dos conjuntos fuzzy, um valor de incerteza é então calculado e associado à resposta do classificador para derivar uma família de métricas classes-específicas com pesos utilizada para comparar imagens com maior precisão. A Figura 4 ilustra o processo de recuperação de imagens da CSWIRe, utilizando uma interface gráfica apropriada.

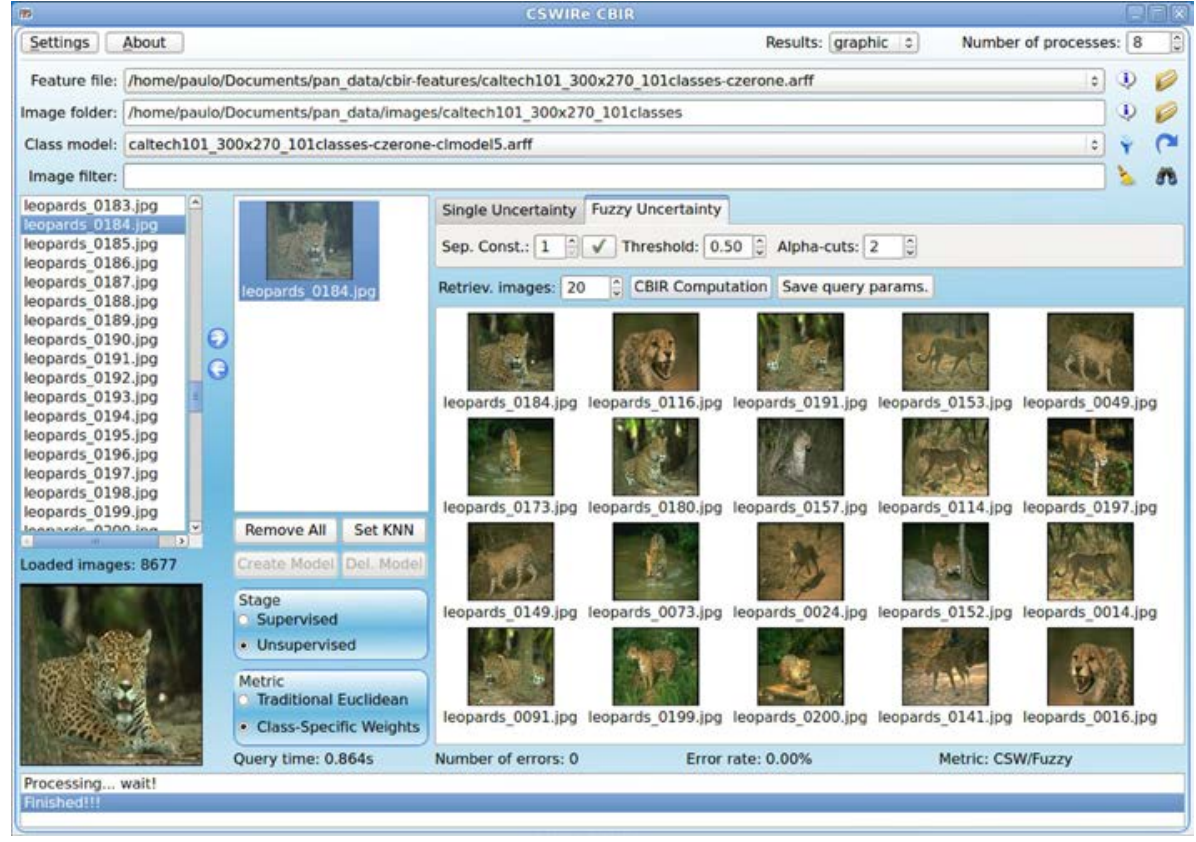

Figura 4. Interface gráfica da CSWIRe, mostrando o processo de recuperação de imagens por conteúdo. 


\section{Outras Produções Relevantes}

Além das contribuições apresentadas anteriormente, merece destaque o trabalho desenvolvido em colaboração com o grupo de pesquisa da Universidade de Calgary ${ }^{1}$, visando a exploração de espaços multidimensionais via projeção inversa, com uso da técnica intitulada inverse-LAMP (iLAMP). Esta técnica executa a projeção inversa através de mapeamentos locais afins que preservam a distância entre as novas amostras de modo preciso, já que ela segue os mesmos preceitos da LAMP. Desse modo, o usuário pode interativamente criar instâncias no conjunto de dados original, gerando assim, dados multidimensionais sintéticos além dos já existentes na disposição inicial. Para maiores detalhes sobre iLAMP, consulte [Dos Santos Amorim et al. 2012].

Para atender os requisitos do projeto, foram desenvolvidas algumas ferramentas computacionais. Projection Analyzer (PAn), um conjunto de bibliotecas de alto desempenho em ANSI C (versão inicial disponível em http://sites.google.com/site/ paulojoiafilho/tools). Também foi implementado um módulo em Python para facilitar a execução das tarefas, que além de reutilizar o código em $\mathrm{C}$, permite integração com pacotes de matemática numérica conhecidos. As interfaces gráficas mostradas nas Figuras 2 e 4, por exemplo, foram desenvolvidas a partir destas ferramentas.

\section{Publicações, Prêmios e Estado da Arte}

A técnica LAMP [Joia et al. 2011a] destaca-se como uma das técnicas de projeção mais precisas da atualidade. O recente trabalho apresentado por [Fadel et al. 2015] comprova este fato ao comparar várias técnicas de projeção (stress e tempo computacional), posicionando a LAMP como uma das técnicas de projeção do estado da arte em relação à preservação de distâncias e custo computacional. Além de um número considerável de citações em trabalhos científicos, LAMP também recebeu menção honrosa na Conferência IEEE Information Visualization, uma das principais dessa área de pesquisa do mundo (mais informações em http: / / www . usp. br/agen/ ?p=79480).

\subsection{Lista de Publicações}

1. [Joia et al. 2011a] "Local Affine Multidimensional Projection", IEEE Transactions on Visualization and Computer Graphics (Qualis A1).

2. [Joia et al. 2011b] "Projection-based Image Retrieval using Class-Specific Metrics", 24th SIBGRAPI, IEEE Computer Society (Qualis B1).

3. [Joia et al. 2012] "Class-Specific Metrics for Multidimensional Data Projection Applied to CBIR", The Visual Computer Journal (Qualis B1).

4. [Dos Santos Amorim et al. 2012] "iLAMP: Exploring High-Dimensional Spacing through Backward Multidimensional Projection”, IEEE VAST’12 (Qualis B1).

5. [Casaca et al. 2013] "Spectral Image Segmentation Using Image Decomposition and Inner Product-Based Metric", Journal of Mathematical Imaging and Vision (Qualis A2).

6. [Joia et al. 2015] "Uncovering Representative Groups in Multidimensional Projections", Computer Graphics Forum (EuroVis'15) (Qualis A2).

7. Joia, P.; da Silva, S. F.; Batista, J.; Nonato, L. G. "Class-specific metrics with weights and uncertainty modeling using fuzzy sets applied to content-based image retrieval", Expert Systems with Applications, Elsevier. Submetido, em processo de revisão (Qualis A1).

\footnotetext{
${ }^{1}$ Interactive Reservoir Modeling and Visualization Group, Universidade de Calgary, Alberta, Canadá.
} 


\subsection{Prêmios}

1. [Joia et al. 2011a] Local Affine Multidimensional Projection. Honorable Mention Award - IEEE InfoVis 2011 (http: / /www.cad.z ju.edu.cn/home/vag/ cwf/ vispapers/infovis2011.html).

2. [Joia et al. 2011b] Projection-based Image Retrieval using Class-Specific Metrics. Best Paper Award - Sibgrapi 2011 (https://www.computer.org/csdl/ proceedings/sibgrapi/2011/4548/00/4548z022.pdf).

\section{Conclusões}

Este trabalho apresentou soluções para problemas de identificação de agrupamentos e busca por similaridade em dados multidimensionais, utilizando técnicas de projeção. Para cada problema, duas novas técnicas foram desenvolvidas, atingindo resultados expressivos em cada uma delas, uma das quais, inclusive, equipara-se ao estado da arte em termos de precisão e eficiência computacional (LAMP).

\section{Agradecimentos}

Agradecemos ao Instituto de Ciências Matemáticas e de Computação (ICMC/USP) pelo suporte ao projeto e à Fundação de Amparo à Pesquisa do Estado de São Paulo (FAPESP) pela apoio financeiro (Processo \#2010/07367-9).

\section{Referências}

Casaca, W., Paiva, A., Gomez-Nieto, E., Joia, P., and Nonato, L. G. (2013). Spectral Image Segmentation Using Image Decomposition and Inner Product-Based Metric. Journal of Mathematical Imaging and Vision, 45(3):227-238.

Dos Santos Amorim, E. P., Brazil, E. V., Daniels, J., Joia, P., Nonato, L. G., and Sousa, M. C. (2012). iLAMP: Exploring High-Dimensional Spacing through Backward Multidimensional Projection. In IEEE Conference on Visual Analytics Science and Technology (VAST), pages 53-62. IEEE.

Fadel, S. G., Fatore, F. M., Duarte, F. S. L. G., and Paulovich, F. V. (2015). LoCH: A neighborhood-based multidimensional projection technique for high-dimensional sparse spaces. Neurocomputing, 150(Part B):546-556.

Joia, P. (2015). Técnicas de projeção para identificação de grupos e comparação de dados multidimensionais usando diferentes medidas de similaridade. Tese de Doutorado, Universidade de São Paulo - USP, São Carlos, SP. Disp. em: <http: / /www.teses.usp.br/teses / disponiveis/55/55134/tde-29032016-143247>. Acesso em: 25 maio 2016.

Joia, P., Coimbra, D., Cuminato, J. A., Paulovich, F. V., and Nonato, L. G. (2011a). Local Affine Multidimensional Projection. IEEE Transactions on Visualization and Computer Graphics, 17(12):2563-2571.

Joia, P., Gomez-Nieto, E., Batista Neto, J., Casaca, W., Botelho, G., Paiva, A., and Gustavo Nonato, L. (2012). Class-specific metrics for multidimensional data projection applied to CBIR. The Visual Computer, 28(10):1027-1037.

Joia, P., Gomez Nieto, E., Botelho, G., Batista Neto, J., Paiva, A., and Nonato, L. G. (2011b). Projection-based Image Retrieval using Class-Specific Metrics. In Lewiner, T. and Torres, R., editors, 24th SIBGRAPI, pages 125-132, Maceio, AL. IEEE Computer Society.

Joia, P., Petronetto, F., and Nonato, L. G. (2015). Uncovering Representative Groups in Multidimensional Projections. Computer Graphics Forum, 34(3):281-290. 